\title{
ON A STOCHASTIC INVENTORY MODEL WITH DETERIORATING ITEMS
}

\author{
L. AGGOUN, L. BENKHEROUF, and L. TADJ
}

(Received 13 April 1999)

\begin{abstract}
We suggest a new inventory continuous time stochastic model for deteriorating items. We derive optimal operating characteristics of the expected cost per unit time under the assumption that demand in each replenishment cycle forms a regenerative process. We also present numerical examples.
\end{abstract}

2000 Mathematics Subject Classification. Primary 60K30, 60J10, 90B05.

1. Introduction. Inventory models for perishable or deteriorating items are of considerable importance in the study of inventory systems. There is an abundant amount of research papers dealing with such models: see Raafat [8] for his excellent review and references therein. Unfortunately, a large proportion of existing work is related to deterministic models. A very limited number of papers on continuous time review models for deteriorating items can be found. Further, there is a very small difference among these models. They are based on a fixed review period of length, say, $T$. At the beginning of each period the inventory level is reset to some level, say, $S$ regardless of the position of the inventory. The aggregate demand is assumed random but uniformly distributed over the period. The perishability process acts deterministically on the stock. The analysis of these models is carried out in a similar fashion to deterministic models: see [2, 3, 4, 5, 9].

It is clear that these models may call for ordering very small quantities with positive probability because of the uniform assumption. This may be very costly.

Nahmias and Shah [7] investigated the problem of finding the optimal lot size reorder point for an extended model of the work mentioned above by considering the possibility of lead time. However, they made a strong assumption that demand is on average fixed per unit time. Also, the deterioration rate was taken to be fixed.

Nahmias and Shah [7] pointed to the difficulty of obtaining the optimal policy, which minimizes the total cost of inventory per unit time, for continuous review models with positive lead time. It is well known that for models with no deterioration the optimal policy is of the form: when the inventory position hits the reorder point $r$, an order for $Q$ units is placed. This is no longer true when deterioration is included.

It is worth mentioning at this stage the pioneering work of Nahmias in the study of discrete time inventory systems for perishable items: see [6] for more details. In these models demand was assumed random in each period and products were assumed to have a certain life time which may be random. Various optimal characteristics were obtained under various conditions on the demand and the life time processes. 
In the present paper, we assume that both demand and deterioration are continuous time stochastic processes. We also include the possibility of random lead time and make the usual assumption that orders are assumed not to cross each other. The model to be presented is inspired from pathwise analysis of stochastic calculus. Here, we assume that the level of inventory $I(t)$ is modelled by a finite family of differential equations each of which corresponds to a possible path to which is attached a probability. Our approach shall be simple and at times heuristic. More sophisticated approach is the subject of future work.

The next section is concerned with the mathematical model and some results. Section 3 contains the results of a numerical study and a conclusion.

2. Model formulation and results. (1) A single item is held in stock.

(2) There is a lead time $L$ which is assumed to be random with density function $f$. We also assume that orders do not cross each other.

(3) Shortages are allowed.

(4) At the beginning of each cycle the decision maker has $N$ possible scenarios of demand-deterioration $\left\{D_{j}(t), \theta_{j}(t)\right\}$ rates that may occur. To each scenario $j$ is attached a probability $p_{j}$, where $\sum_{j=1}^{N} p_{j}=1$.

(5) The demand rates $D_{j}(t)$ and the deterioration rates $\theta_{j}(t)$ are a function of the length of the cycle. This assumption fits well with products experiencing some kind of quality changes while in stock such as food stuff, batteries, electronic components, cars, computers, etc. These products usually experience a decrease in demand. However, there are products that experience an increase in demand while in stock: some types of drinks and cheese, antiques, etc.

(6) The cost structure of the model is as follows:

(a) a fixed ordering cost, $K$,

(b) a holding cost per unit of item per unit time $c_{1}$,

(c) a shortage cost per unit of item per unit time $c_{2}$,

(d) a cost per unit of item $c_{3}$.

Assume that at time $t=0$, the system has a quantity of $Q$ units in stock. The quantity depletes due to demand and deterioration according to one of the $N$ possible scenarios until it reaches level $r$ at which time an order to replenish the inventory to level $Q$ is made. Once the order arrives a new cycle begins.

Note that during the lead time shortages may occur.

Let $T_{i}$ be the elapsed time between the $i$ th and the $(i+1)$ th cycle, with $T_{0}=0$. Also, let $S_{n}=\sum_{i=1}^{n} T_{i}$. Obviously, under assumptions 4 and $5, T_{i}$ are i.i.d. Also, it is easy to see that the sequence $S=\left\{S_{n}, n=1, \ldots\right\}$ forms a renewal process. Standard results from the theory of renewal reward processes enable us to write down the total cost per unit time TCU as:

$$
\mathrm{TCU}=\frac{E[\text { cost for the first cycle }]}{E[\text { length of the cycle }]} .
$$

Our aim now is to write down explicitly the expression (2.1). 
Let $I(t)$ be the level of inventory at time $t$, where $I(0)=Q, T$ be the length of the first cycle, say, and let

$$
I^{+}(t)=\left\{\begin{array}{ll}
I(t) & \text { for } I(t) \geq 0, \\
0 & \text { for } I(t)<0,
\end{array} \quad I^{-}(t)= \begin{cases}0 & \text { for } I(t) \geq 0, \\
I(t) & \text { for } I(t)<0 .\end{cases}\right.
$$

It follows that (2.1) gives

$$
\mathrm{TCU}=\frac{K+E\left[c_{1} \int_{0}^{T} I^{+}(t) d t+c_{2} \int_{0}^{T} I^{-}(t) d t+c_{3} P\right]}{E[T]} .
$$

Here $P$ refers to the quantity of perished items during the cycle. We are interested in finding the values of $Q$ and $r$ that minimizes TCU in (2.3).

Generally speaking, if we let the level of inventory, $I(t)$, be modelled by a general stochastic differential equation, then finding the values of $Q$ and $r$ that minimizes (2.3) becomes a formidable task involving techniques and tools from stochastic calculus. This more sophisticated approach shall be discussed in future work. In this paper, we shall adopt a direct approach. In this case, we need only to look at each realization of the process on each possible path. This usually reduces to a deterministic analysis on each path. Then, we take expectation over these paths. We hope that this paper will open a way for the use of powerful tools for solving still a large number of open problems.

For a given $j$ and $L, j=1, \ldots, N$, let $I_{j}^{L}(t)$ be the level of inventory at time $t$ given that the process is described by $\left\{D_{j}(t), \theta_{j}(t)\right\}$ when the lead time is $L$. Also, let $T_{j}^{L}$ be the length of the cycle and let $\Delta_{j}^{L}$ be the time taken for the inventory to reach level zero starting from level $Q$.

Write $t_{j}$ for the time needed for the inventory to reach level $r$ starting from $Q$. Also, assume that the functions $D_{j}(t)$ and $\theta_{j}(t)$ are smooth for all $j=1, \ldots, n$, meaning that they are differentiable whenever needed.

Let $\tau_{j}^{L}=\min \left(\Delta_{j}^{L}, t_{j}+L\right)$. Then, the variation of $I_{j}^{L}(t)$ with respect to time can be shown to be governed by the following differential equation:

$$
\frac{d I_{j}^{L}(t)}{d t}=-D_{j}(t)-\theta_{j}(t) I_{j}^{L}(t), \quad 0 \leq t<\tau_{j}^{L}
$$

with boundary condition $I\left(\Delta_{j}\right)=0$. Also, if $\tau_{j}^{L}<t_{j}+L$, then shortages occur, in which case $\tau_{j}=\Delta_{j}$ and the changes in the inventory after time $\tau_{j}^{L}$ and up to $t_{j}+L$ is governed by the following differential equation:

$$
\frac{d I_{j}^{L}(t)}{d t}=-D_{j}(t), \quad \Delta_{j}^{L} \leq t<t_{j}+L,
$$

with initial condition $I\left(\Delta_{j}\right)=0$.

Note that in (2.5) the deterioration rate $\theta_{j}(t)$ is missing. This is simply due to the fact that during shortages no deterioration is experienced.

Direct calculations show that the solution to (2.4) may be represented by

$$
I_{j}^{L}(t)=e^{-g_{j}(t)} \int_{t}^{\Delta_{j}} e^{g_{j}(u)} D_{j}(u) d u,
$$


with

$$
g_{j}^{\prime}(t)=\theta_{j}(t)
$$

and $g_{j}(0)=0$. Here $g^{\prime}(\cdot)$ represents the first derivative of $g$ with respect to its argument.

Now, it is not difficult to deduce that

$$
\begin{gathered}
Q=\int_{0}^{\Delta_{j}} e^{g_{j}(t)} D_{j}(t) d t \\
r=e^{-g_{j}\left(t_{j}\right)} \int_{t_{j}}^{\Delta_{j}} e^{g_{j}(t)} D_{j}(t) d t .
\end{gathered}
$$

It is clear that there is one-to-one correspondence between $Q$ and $\Delta_{j}$ since $e^{g_{j}(t)} D_{j}(t)$ $>0$. Also, if $\Delta_{j}$ is known, then there is a one-to-one correspondence between $r$ and $t_{j}$.

The amount of inventory $A_{j}^{L}$ during the cycle is equal to

$$
A_{j}^{L}=\int_{0}^{\tau_{j}^{L}} I_{j}^{L}(t) d t
$$

where $I_{j}^{L}(t)$ is given by (2.6). When $\theta_{j}(t)=\theta_{j}$ then it can be shown that (2.9) reduces to

$$
\frac{1}{\theta_{j}} \int_{0}^{\Delta_{j}}\left(e^{\theta_{j} t}-1\right) D_{j}(t) d t-\frac{1}{\theta_{j}} \int_{\tau_{j}^{L}}^{\Delta_{j}}\left(e^{\theta\left(u-\tau_{j}^{L}\right)}-1\right) D_{j}(t) d t .
$$

Also, the amount of shortages $S_{j}^{L}$ can be shown to be equal to

$$
S_{j}^{L}=\int_{\tau_{j}^{L}}^{t_{j}+L}\left(t_{j}+L-t\right) D_{j}(t) d t .
$$

It is worth mentioning at this stage that relations (2.11) and (2.12) reduce to their deterministic counterpart model when $L=0$ since $\Delta_{j}=\tau_{j}^{L}$ (see [1]).

The amount of deteriorated items $P_{j}^{L}$ during the cycle is

$$
P_{j}^{L}=Q-I_{j}^{L}\left(\tau_{j}^{L}\right)-\int_{0}^{\tau_{j}^{L}} D_{j}(t) d t
$$

Where $I_{j}^{L}(t)$ is given by (2.6).

When $\theta_{j}(t)=\theta_{j}$, then (2.13) reduces to

$$
P_{j}^{L}=\int_{0}^{\tau_{j}^{L}}\left(e^{\theta_{j} t}-1\right) D_{j}(t) d t+\int_{\tau_{j}^{L}}^{\Delta_{j}} e^{\theta_{j} t}\left(1-e^{-\theta_{j} \tau_{j}^{L}}\right) D_{j}(t) d t .
$$

Note that in this case we have

$$
P_{j}^{L}=\theta_{j} A_{j}^{L}
$$

Now, let

$$
T C_{j}^{L}=K+c_{1} A_{j}^{L}+c_{2} S_{j}^{L}+c_{3} P_{j}^{L},
$$

where $A_{j}^{L}, S_{j}^{L}$, and $P_{j}^{L}$ are given by (2.10), (2.12), and (2.13), respectively. 
It follows from (2.3) that

$$
\mathrm{TCU}=\frac{\sum_{j=1}^{N} \int_{0}^{\infty} p_{j} T C_{j}^{L} f(L) d L}{\sum_{j=1}^{N} \int_{0}^{\infty} p_{j} T_{j}^{L} f(L) d L},
$$

where $T C_{j}^{L}$ is given by (2.16).

We deduce from the previous analysis that the problem of finding the optimal replenishment strategy reduces to the problem of finding $Q$ and $r$ which minimizes TCU in (2.17) subject to (2.8) and (2.9).

REMARK. Fix $r=0$ and assume that $D_{j}(t)=\lambda_{j}$ and $\theta_{j}(t)=\theta_{j}, j=1, \ldots, n$. Also, put $L=0$, then (2.16) reduces to

$$
T C_{j}^{L}=K+\frac{c_{1}+\theta_{j} c_{3}}{\theta_{j}} \int_{0}^{\Delta_{j}^{L}}\left(e^{\theta_{j} t}-1\right) \lambda_{j} d t .
$$

Now, let $\theta_{j} \rightarrow 0$. Then, it is not difficult to deduce, after some algebra, that (2.17) reduces to

$$
\frac{K}{Q \sum_{j=1}^{N}\left(p_{j} / \lambda_{j}\right)}+\frac{1}{2} c_{1} Q
$$

from which the optimal order quantity is

$$
Q=\sqrt{\frac{2 K\left(1 / \sum_{j=1}^{N}\left(p_{j} / \lambda_{j}\right)\right)}{c_{1}} .}
$$

Expression (2.20) means that the economic order quantity is recovered in our model by taking the demand rate to be equal to $1 / \sum_{j=1}^{N}\left(p_{j} / \lambda_{j}\right)$.

3. Numerical examples and conclusions. In this section, we present detailed results of two examples for which the optimal replenishment schedule is based on minimizing (2.17). We also give the result of a small scale computational comparison between the optimal scheme and an approximate scheme based on taking a single scenario whose demand and deterioration rates are assumed to be the weighted averages of the scenarios (see below for more details).

In all our examples, we assumed that the demand rates are linear functions, that is, $D_{j}(t)=a_{j} t+b_{j}, j=1, \ldots, N$, where $N=4$. We took $a_{1}=250, a_{2}=90, a_{3}=80$, $a_{4}=200, b_{1}=40, b_{2}=30, b_{3}=100, b_{4}=50, K=200, c_{1}=0.7, c_{2}=1.3, c_{3}=8.5$. The lead time distribution was assumed to be uniform with support $(0, m)$, where $m$ was fixed to 2.

In the first example, we took $p_{1}=p_{2}=p_{3}=p_{4}=0.25$ and in the second example we took $p_{1}=0.1, p_{2}=0.3, p_{3}=0.4$, and $p_{4}=0.2$.

To find the optimal policy we used a NAG library routine. For the expressions in (2.5) we used the package derive for symbolic integration.

The optimal policy in the first example gave $Q^{*}=20.6677$ and $r^{*}=4.1377$ with total cost per unit time equals 291.9599.

The optimal policy in the second example gave $Q^{*}=26.5956$ and $r^{*}=5.3279$ with total cost per unit time equals 278.9021 . 
Further, we compared the optimal policy based on minimizing (2.17) with an approximate scheme based on taking a single scenario whose demand is the weighted average of the scenarios. To be precise, we consider a single scenario $N=1$ in (2.17) with

$$
D(t)=\sum_{j=1}^{N} p_{j} D_{j}(t), \quad \theta(t)=\sum_{j=1}^{N} p_{j} \theta_{j}(t) .
$$

In our comparison we only considered the case $D_{j}(t)=a_{j} t+b_{j}, j=1, \ldots, 4$. We took eighty problems in which we fixed $a_{1}=250, a_{2}=90, a_{3}=80, a_{4}=200, b_{1}=40$, $b_{2}=30, b_{3}=100, b_{4}=50, K=200, c_{1}=0.7, c_{2}=1.3, c_{3}=8.5$. In the first forty problems, (Problems I), we assumed that $p_{1}=p_{2}=p_{3}=p_{4}=0.25$ and in the remaining forty problems, (Problems II), we put $p_{1}=0.1, p_{2}=0.3, p_{3}=0.4$, and $p_{4}=0.2$.

In all the problems, we varied the set up cost $K=150,160, \ldots, 240$, holding cost, $c_{1}=0.2,0.3, \ldots, 1.1$, shortage cost $c_{2}=0.8,0.9, \ldots, 1.7$, and the unit cost $c_{3}=3.5$, $4.5, \ldots, 12.5$.

The approximate policy was assessed in terms of the percentage deviation from optimality as the percentage increase in the total cost above the optimal cost value.

Tables 3.1 and 3.2 show the average cost deviation of the approximation with respect to each parameters.

TABLE 3.1. Average deviation of Problems I as a function of the parameters $K, c_{1}, c_{2}$, and $c_{3}$.

\begin{tabular}{cccc}
\hline Parameters & Max deviation & Min deviation & Average \\
\hline$K$ & 0.0262 & 0.0204 & 0.02585 \\
$c_{1}$ & 0.0330 & 0.0143 & 0.02199 \\
$c_{2}$ & 0.0420 & 0.0109 & 0.02194 \\
$c_{3}$ & 0.0628 & 0.0099 & 0.02827 \\
\hline Overall average & 0.04100 & 0.01388 & 0.02451 \\
\hline
\end{tabular}

TABLE 3.2. Average deviation of Problems II as a function of the parameters $K, c_{1}, c_{2}$, and $c_{3}$.

\begin{tabular}{cccc}
\hline Parameters & Max deviation & Min deviation & Average \\
\hline$K$ & 0.0162 & 0.0110 & 0.01402 \\
$c_{1}$ & 0.0245 & 0.0054 & 0.01241 \\
$c_{2}$ & 0.0270 & 0.0045 & 0.01505 \\
$c_{3}$ & 0.0325 & 0.0050 & 0.01226 \\
\hline Overall average & 0.02505 & 0.00648 & 0.013435 \\
\hline
\end{tabular}

It is clear that the approximate method performs remarkably well in this case. In the eighty problems, the deviation was less than $0.03 \%$. We think that more computational 
studies with different demand, deterioration rates, functions, and general distribution of the lead time should be undertaken.

To summarize, we have suggested in this paper a new continuous time inventory model for deteriorating items. Optimal $(r, Q)$ policies that minimizes the total cost per unit time were found. Numerical examples with the result of a computational comparison between the optimal schedule and an approximate method were presented.

\section{REFERENCES}

[1] L. Benkherouf and M. G. Mahmoud, On an inventory model for deteriorating items with increasing time-varying demand and shortages, J. Oper. Res. Soc. 47 (1996), no. 1, 188-200. Zbl 842.90027.

[2] M. R. Chowdhury and K. S. Chaudhuri, An order-level inventory model for deteriorating items with finite rate of replenishment, Opsearch 20 (1983), 99-106. Zbl 521.90042.

[3] U. Dave and M. C. Jaiswal, A discrete in-time probabilistic inventory model for deteriorating items, Dec. Sci. 11 (1980), 110-120.

[4] U. Dave and Y. K. Shah, A probabilistic inventory model for deteriorating items with lead time equal to one scheduling period discrete in-time probabilistic inventory model for deteriorating items, European J. Oper. Res. 9 (1978), 281-285.

[5] B. B. Jani, M. C. Jaiswal, and Y. K. Shah, $\left(S, q_{p}\right)$ system inventory model for deteriorating items, Int. J. Prod. Res. 16 (1978), 1-9.

[6] S. Nahmias, Perishable inventory theory: A review, Oper. Res. 30 (1982), 680-708. Zbl 486.90033 .

[7] S. Nahmias and S. W. Shah, A Heuristic lot size reorder point model for decaying inventories, Mgmt. Sci. 25 (1979), 90-97.

[8] F. Raafat, Survey of literature on continuously deteriorating inventory models, J. Oper. Res. Soc. 42 (1991), no. 1, 27-37. Zbl 718.90025.

[9] Y. K. Shah and M. C. Jaiswal, An order-level inventory model for a system with constant rate of deterioration, Opsearch 14 (1977), no. 3, 174-184. MR 56\#14634.

L. Aggoun and L. Benkherouf: Department of Mathematics and Statistics, Sultan QABoos University, P.O. Box 36, AL-KHOD 123, Sultanate OF OMAN

L. TADJ: Department of Mathematics and Operations Research, King SAUd UniverSITY, P.O. BOX 2455, RIYADH 11451, SAUDI ARABIA

E-mail address: 1otftadj@ksu.edu.sa 




Advances in

Operations Research

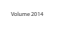

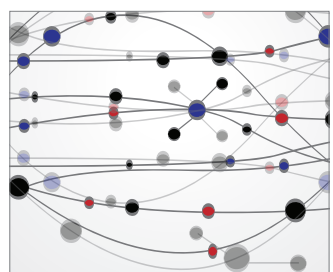

\section{The Scientific} World Journal
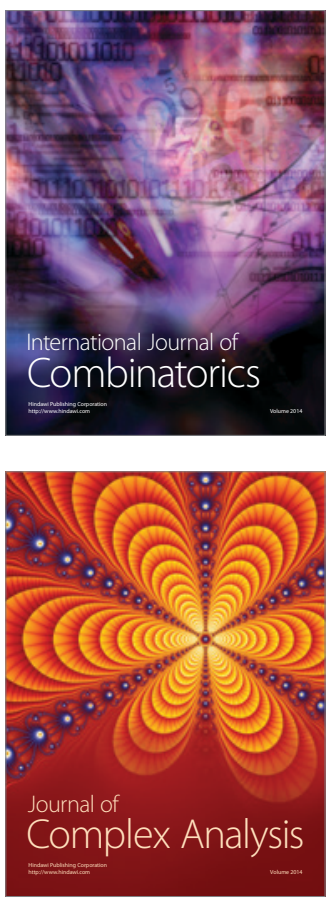

International Journal of

Mathematics and

Mathematical

Sciences
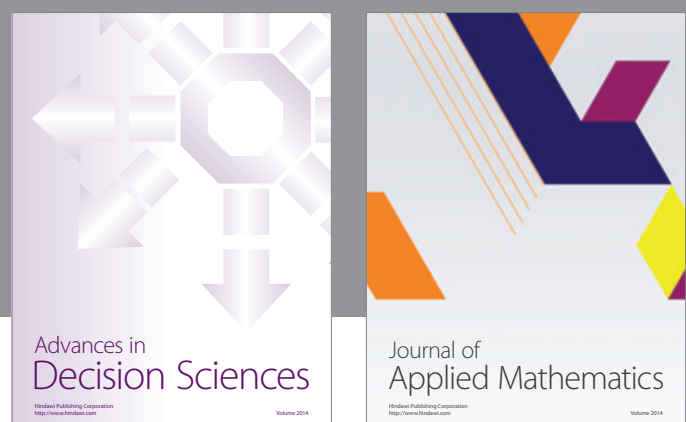

Journal of

Applied Mathematics
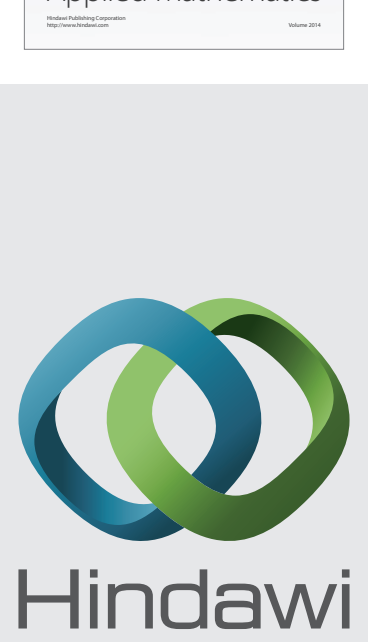

Submit your manuscripts at http://www.hindawi.com
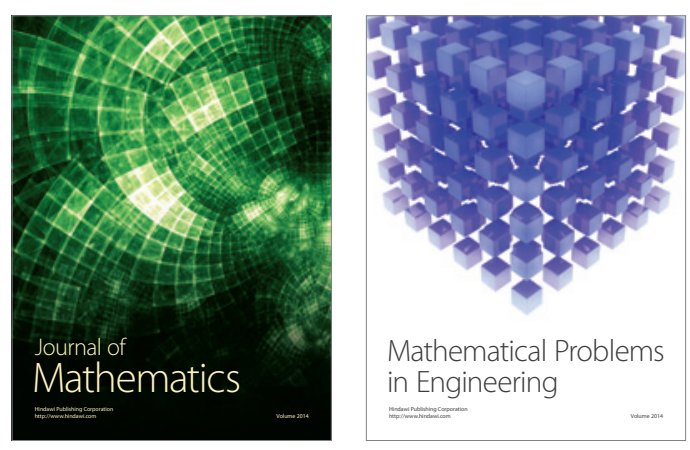

Mathematical Problems in Engineering


Journal of

Function Spaces




ournal of

Probability and Statistics

Promensencen
